\title{
Time and Geology in Geographic Information Systems: Some Formalism for Describing Temporal Events
}

\author{
Stephan van Gasselt ${ }^{1}$ and Andrea Nass ${ }^{2}$ \\ ${ }^{1}$ Institute of Geological Sciences, Freie Universitaet Berlin, Germany · \\ stephan.vangasselt@fu-berlin.de \\ ${ }^{2}$ Institute of Planetary Research, German Aerospace Center (DLR), Berlin/Germany \\ Full paper double blind review
}

\begin{abstract}
Since first concepts and prototypes for integrated geographic information systems (GIS) have been designed, incorporation of time has always been a matter of research, philosophical debate, and development of innovative ideas. Commercially available informationsystem packages usually do not provide support for time integration in a satisfactory way, as their design follows a cartographic 2.5D-model approach. This shortcoming is particularly problematic when designing GIS data models for geologic (map) data, which are spatially $3 \mathrm{D}$, and - by definition - temporal, as geology is all about connecting time and space to physical units. Despite its time-relevance, classical GIS-based geologic data models cannot implement time and temporal information, which is usually hard-wired into relations as attribute values. Consequently, and due to this structural problem, data integrity can be at risk, time queries are not possible, and the full potential of working with geologic map data cannot be unlocked. We herein discuss basic ontologic concepts of time in geology, and incorporate these aspects in order to provide ideas and potential solutions of approaching time in GIS-integrated data models.
\end{abstract}

\section{Aims of Study}

GIS data models are paramount for efficiently maintaining integrity, administrating contents and querying/analyzing data, which are undergoing regular updates and changes in the course of projects. In geologic sciences, data models should allow the integration of (a) spatial data, i.e., geometries in connection with their geologic attributes, as well as their (b) temporal reference, i.e., timing of geologic events, for purposes such as analysis and map production.

Our work is focused on establishing a generic time-integrated data model for geologic mapping and generation of map output. Parts of this work have led to discussions on the nature of time integration and to conceptualizations with respect to the treatment of time inherently related to geologic units, and, thus, geologic map products. 
This contribution outlines the nature and relevance of time in geology and geologic mapping by discussing different time scenarios which are, hopefully, also applicable to other research fields.

\section{Ontological Aspects of Geologic Time}

Nowadays, geologic data objects are organized and analyzed in an integrated way within modern Geographic Information Systems (GISs), in connection with spatial databasemanagement system (DBMS) technology. Such systems usually provide means for managing, analyzing, and providing a map-projected, two-dimensional thematic view of $2.5 \mathrm{D}$ or $2+1 \mathrm{D}$ data, by establishing a common geometric reference. With respect to attribute dimension of geospatial data, modern GIS and geospatial DBMS do not usually have any limitations, which allows the efficient performance of complex data queries and analyses across all thematic (attribute) dimensions and object layers.

However, GIS-based data integration and analyses experience two major challenges that are related to incorporation and modeling of higher dimensions, which are not explicitly covered on the attribute level: (true) space and time. Geographic, and, in particular, geologic data are always related to the third spatial dimension, and are also always related to time (e.g., THRIFT 1977) as every piece of data is valid only at a discrete instance or during a defined interval. The retrieval of data representing the state of knowledge at a given time is straightforward, as this usually represents the state of data implementation (also called database time). Unfortunately, however, it does not allow the user to query and compare data gathered at or during a different point or period in time. In a changing environment, such queries are not only important for civil-engineering purposes, or planning in a variety of application fields, but they also become important in the field of fundamental research topics related to geomorphology and geology.

The highlights we have set should point towards major issues of an otherwise highly complex topic that has been debated since the 1960 s, and which has been discussed very recently in COX \& RICHARD (2015).

Time in geography and congeneric disciplines can be considered in different ways, as it is either a linear continuum without a distinct beginning and an end, or it may be represented by discrete steps at pre-defined time-resolution intervals. An exhaustive discussion on this topic is given in classic work by THRIFT (1977), PRED (1977), LANGRAN (1992), PEUQUET (1999), OTT \& SWIACZNY (2001), WACHOWICZ (2003), RAPER (2005), LE \& USERY (2009) or COX \& RICHARD (2015). Independent of the approach and understanding of time and its implementation, questions concerned with time-relevant studies can usually be reduced to three query types: (1) queries for a discrete time $t_{i}$ at which a process initiated/terminated, or at which an event has occurred, (2) queries for time ranges/intervals $t_{j}-t_{i}$ during which a number of events and changes have occurred, or (3) queries for rates of changes $\mathrm{d} x / \mathrm{d} t$ of a spatially dependent variable $x$. In the same way, queries can be reversed by querying for events at a given time. In the field of classic geology, and, in particular, for geologic mapping, time is associated with each spatial entity, either implicitly or explicitly, due to the fact that geology is constrained and defined by time (see, e.g., Gould 1987). Such a definition of time is often represented as a spiral of time, i.e., the general perception of geologic time is given by an irreversible continuum (e.g., NACSN 2005) with a starting point indi- 
cating planet formation and a terminal point representing the presence. Thus, geologic time is usually linear and absolute in nature (CLARAMUNT \& THERIAULT 1995, LE \& USERY 2009) and contrasts the concept of cyclic or periodic time.

Geologic time, i.e., the general geochronology, is - as internationally defined - partitioned into successively smaller levels of time units with distinct boundaries, defined by Global Boundary Stratotype Sections Ages (GSSAs). This concept of time is absolute, and thus measurable (chronometric time), with subdivisions termed eons on the level of $10^{9}$ years, eras, periods, epochs, and, finally, ages (see figure 1) as well as SALVADOR (1994), GRADSTEIN et al. (2012), COUVERING \& OGG (2007), OGG et al. (2008) and references cited therein. This leads to another distinct feature of geologic time: time is measured backwards as geologic events and changes (boundaries) occurred before present time. Consequently, geologic boundaries are dynamic with respect to today and static with respect to a predefined reference date.

In Geology, the relationship between spatial location and time has been established in the $17^{\text {th }}$ century when it was discovered that the vertical stack of rocks is a measure of time at which the oldest rock unit - when undisturbed - is covered up by more recent rock units (STENO 1917), and each rock unit of a surface can be associated with a formation age. An explicit time assignment is provided if direct measurements of a particular surface unit via, e.g., radiometric methods, are available. An implicit time assignment is more involved as each geologic environment has its very own boundary conditions and its very own characteristic materials deposited at a given time.

The link between materials deposited at two ends of the world is only given by the chronometric time, which puts these units into two chronological boundaries. A rock unit is therefore not only characterized by its material, but also by its time, and therefore has a stratigraphic code used in a local/regional context (chronostratic time). For chronostratigraphy, material units rather than time units are employed and placed into stratigraphic boundaries: eonothems, erathems, periods, systems and series as chronostratigraphic counterparts of the aforementioned chronologic boundaries. International approaches are successfully working on homogenizing local stratigraphic flavors and putting units into an international context. Still, old maps and categorizations do and will always exist, and thus they must be treated appropriately within a data model employed in an integrated system. This earth-oriented approach has been transferred to all solid-surface planetary objects in the 1950s to 1960s, mainly by the United States Geological Survey (USGS). Methods of age measurements and defining stratigraphic boundaries vary, but the baseline remains essentially the same (SALVADOR 1994, OGG et al. 2008).

All pieces of information put on a cartographic product such as a geologic map targets at depicting two issues at the same time: (1) the spatial extent of materials and rock units exposed at the surface, and (2) the temporal extent with respect to the chronostratigraphic context by relating spatial units to an age. Usually, this is achieved by color-coding techniques and an associated map legend.

Without a legend, the stratigraphic relationships, i.e., the sequence of material deposition and emplacement, can only be geometrically reconstructed from the spatial relationships of map units with respect to their topographic relationships. Thus, the need for time integration lies in the nature of geologic mapping and time attributes establish a link for answering questions concerned with (1) unit emplacement time, (2) correlation between dif- 
ferent units, and (3) attributes of geologic units. In the same way, a data model depicting time relationships must also provide means for inverting such queries.

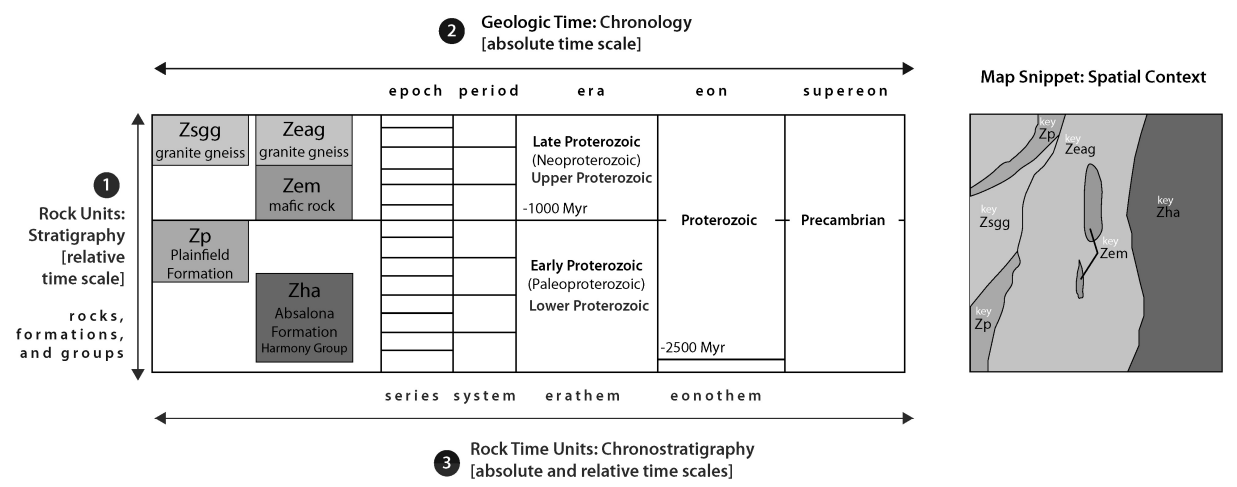

Fig. 1: Representation of geologic time on a (1) relative scale by the superposition of rocks, (2) by the (geo-) chronology on an absolute scale measured backwards from today, and (3) by the chronostratigraphy that combines (1) and (2).

Finally, we arrive at three distinct levels of time: (1) different levels of a reversely organized continuum of absolute and relative geologic time, (2) a continuum of absolute validity time at which geologic times were considered correct, (3) a continuum of absolute transaction time at which data were stored in the database. We here focus on the storage and query of geologic time; transaction time has been dealt with in some detail by, e.g., DATE (2003, 59 f.).

For geologic mapping, a number of agencies and institutions have been working on data models in order to combine and homogenize their institutional or national map portfolio. One of the most prominent examples is The National Geologic Map Database Project (NGMDB) (SOLLER \& BERG 2003, SOlLER \& STAMM 2008), which is based upon the core structure and new developments of the North American Geologic Map Data Model (NADM). This all-encompassing effort provides data models that allow to describe and query stratigraphic codes and stratigraphic relationships.

Ages are, however, usually hard-coded into descriptive terms and thus it is not possible to use different age models at the same time or to query chronologic data. Data integrity is usually controlled by using predefined coded-value domains of mixed entries and dedicated subtypes. Unfortunately, such approaches are highly specific with respect to the design, and a database migration to a different DBMS requires updating the overall implementation (see also COX \& RICHARD 2015).

\section{Towards a Generic Time-Integrative Data Model}

Our sandbox data model has been physically implemented using ESRI's commercial software suite ArcGIS, using its file-based geodatabase model (FGDB), as well as Microsoft's Server Relational Database Management System (RDBMS) with an ArcSDE connector. 
Though easily transportable and implementable on stand-alone architectures via the Extensible Markup Language (XML) Interchange format (XMI), options for querying filegeodatabases from the main interface are limited. The logical design thus differs from the conceptual layout presented here. During design we focused on two low-level requirements: (1) co-existence of different time standards to allow adaptation, and (2) implementation of the concepts of relative and absolute time as discussed above.

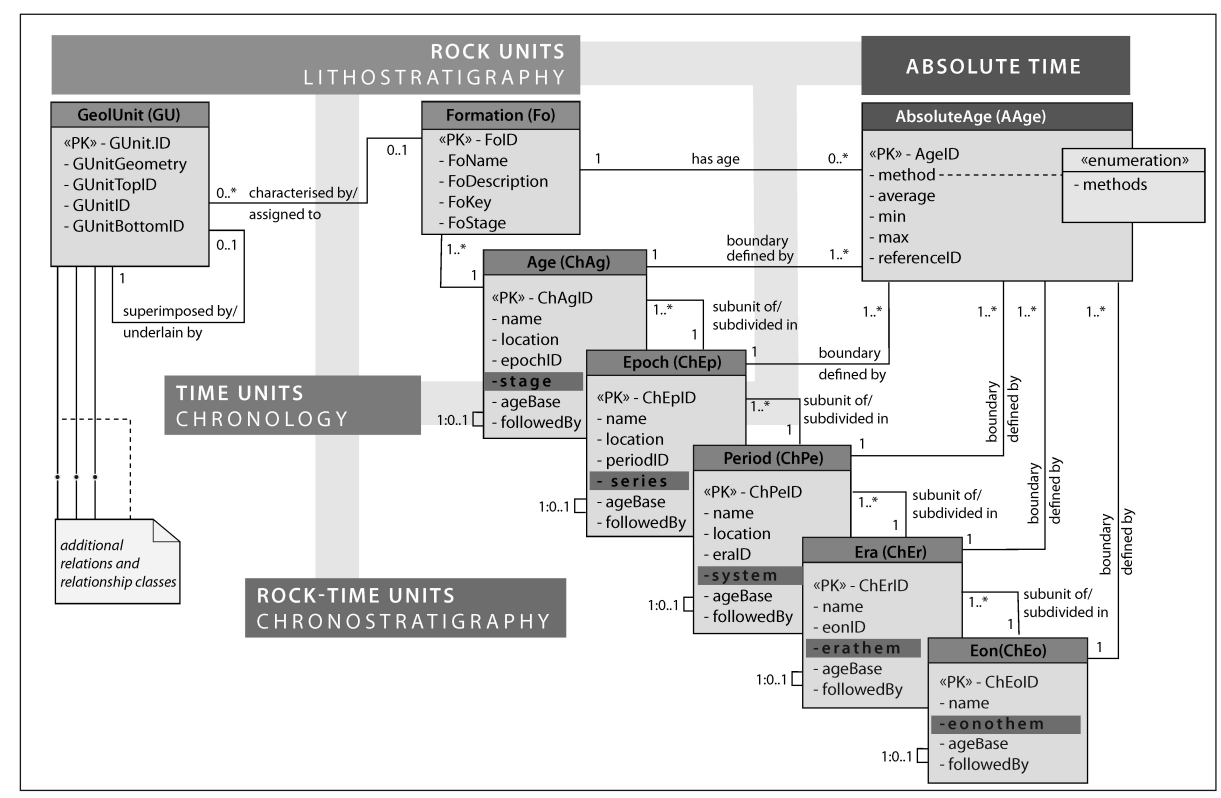

Fig. 2: A geologic-time data model that accounts for relative and absolute time queries and management of different time definitions. Relations in red colour refer to absolute time; brownish-colourised relations refer to geometries and relative stratigraphic time.

The overall design is developed around the geometry feature class GeolUnit (Gu) representing the entity of a mapped geological unit (figure 2). Attributes, such as rock types and descriptions, as well as ancillary information, are transferred to other relations joined by m:n-relationship classes. In this context, so-called formation-attributes around which the chronology section is developed become important. Relationships between formations or lithological units to a position on the time scale are provided by a set of hierarchically organized cascading relations dealing with chronostratigraphy (systems/series). These reference chronologic units (epochs/periods) are defined through a published specification or standard (figure 2). All ages and age ranges are determined by an age relation (A) in which not only ages but also references are stored (implicit concept). Though simple in terms of base design, the model allows accessing the concept of geologic ages from different viewpoints, as outlined in the discussion. Atomizing age information not only allows new geologic chronology models to be easily integrated, but also the easy update of existing attribute values without invoking anomalies. The following scenarios will provide a selection of 
queries and data model return using different time-related queries. First, the general relational algebraic expression is given ( $\pi$ denotes a projection, $\sigma$ is a selection, $\otimes$ denotes a join), followed by the SQL statement. Attribute names and relations refer to names in figure 2.

Generic Time-Step Boundaries: Discrete chronostratigraphic time steps (boundaries) for each subunit are given by a recursive join for each stratigraphic relation. The result is a relation providing (chrono-) stratigraphic units and their time boundaries for chronologic or chronostratigraphic units for, e.g., epochs or series, respectively:

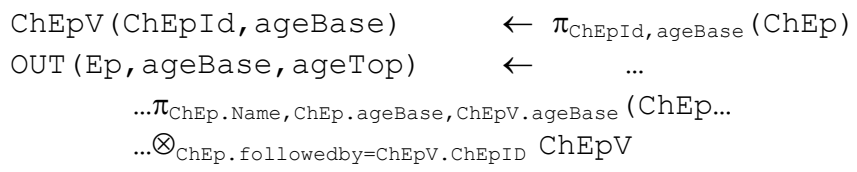

This expression depicts a procedural approach required for interactively querying data; it translates to the following SQL query suitable for DBMS interfaces.

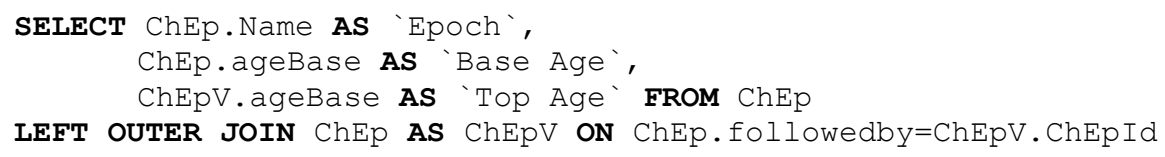

Hierarchical Stratigraphic Relationships: Chronostratigraphic subdivisions are obtained using a cascading right outer join. The given example descends from the stratigraphic period-level downwards, and can be easily extended using higher-level subdivisions, i.e., epochs and ages, in the same way.

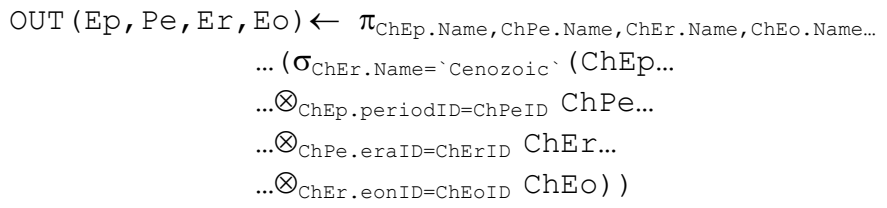

This query returns an output resembling a stratigraphic table and can easily be combined using age boundaries, as shown in the first example. It also allows querying local stratigraphic naming conventions at higher levels, so that results can directly be related to mapunit geometries as shown below.

SELECT ChEp.Name AS `Epoch`,

ChPe.Name AS 'Period',

ChEr.Name AS 'Era',

ChEo.Name AS 'Eon'

FROM ChEp

RIGHT OUTER JOIN ChPe ON ChEp.periodID=ChPeID

RIGHT OUTER JOIN ChEr ON ChPe.eraID=ChErID

RIGHT OUTER JOIN ChEo ON ChEr.eonID=ChEoID

WHERE ChEr.Name="Cenozoic"

ORDER BY ChEr.AgeBase DESC,

ChPe.AgeBase DESC,

ChEp.AgeBase 
Emplacement Time of a Unit: The time geologic units were emplaced, returning an absolute chronostratigraphic or relative time. Such a query involves a relationship between attributes in relation GeolUnit (GU) as depicted in figure 2.

Unit names and associated vocabulary, as well as descriptions, are provided through a relationship to relation Formation (FO). Descriptive elements and symbol keys stored as attribute values in Fo could practically be applied to legend and map layout. Primary keys of geometries stored in the relation $\mathrm{Gu}$, as well of formations ( $\mathrm{FO}$ ), are usually assigned by the DBMS; their relationships need to be defined through a foreign key attribute in the geometry relation $\mathrm{Gu}$. The query result optionally provides information on geologic units spatially located below or above the queried unit on the attribute level. The relative position of units (formations) with respect to the overlaying and underlying units rather than stratigraphy is given by

$$
\begin{aligned}
& \text { GuT } \leftarrow \sigma_{*}(\mathrm{FO}) \\
& \text { GuB } \leftarrow \sigma_{*}(\mathrm{FO}) \\
& \text { TForm } \leftarrow \sigma_{*}(\mathrm{FO}) \\
& \text { BForm } \leftarrow \sigma_{*}(\mathrm{FO})
\end{aligned}
$$

where GuT and GuB refer to top and bottom units, respectively, and TForm and BForm refer to stratigraphic formations of the top and bottom unit.

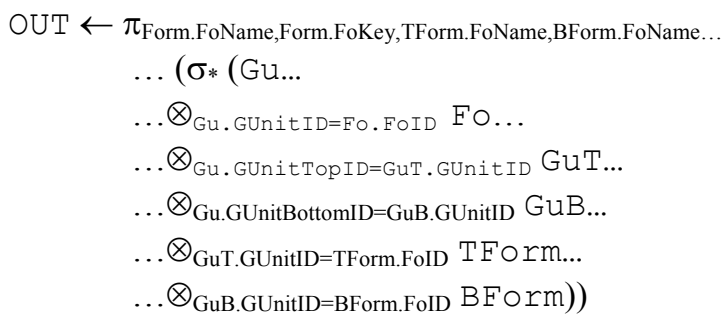

Depending on the DBMS/FGDB interface implementation within the GIS package, a number of views need to be defined before querying. These, again, refer to the top and bottom unit geometries (GuT and GuB) and their geologic attribute values (TForm and BForm).

SELECT

FROM Gu

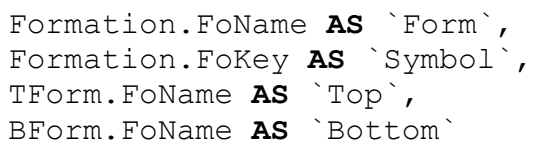

This approach relates the relative emplacement time of a sequence of units identified through their geometry. In order to extract the absolute time of a unit's emplacement, i.e., its age, the geologic unit needs to be related to its absolute age measurement using an additional join. The FoStage attribute contains the chronostratigraphic stages, which refer to the AAge relation. Thereby, a number of different ages with respect to their different mea- 
surement methods can be stored. The following example depicts the identification of a geometry $(\mathrm{Gu})$ related to its geologic unit $(\mathrm{FO})$ and it absolute age (AAge) by returning the name of the geologic formation, its key symbol and its average age (in kiloyears).

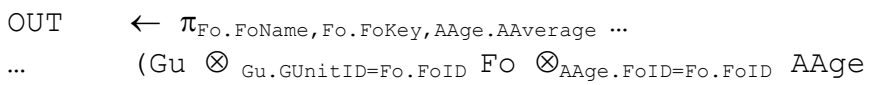

The SQL query reads as follows:

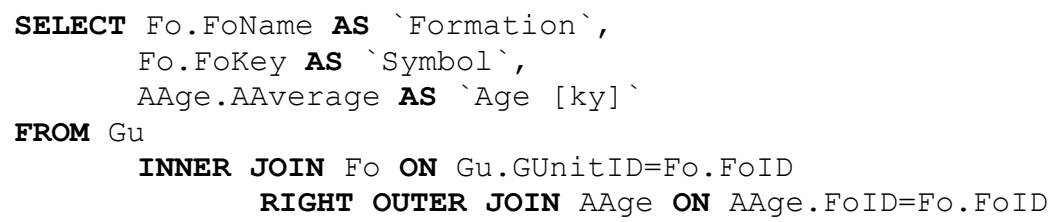

The correlation of time-units between different spatial objects can be subdivided into queries that are (a) related to the same spatial domain, and (b) to those that are part of different spatial domains, i.e., different objects. Both query types relate geometric units to time in the way that a distinct time or time-interval is queried, and all geometric entities are returned as results. The solution of querying entities within the same spatial domain is straightforward, as shown below. For different spatial domains such an issue becomes more complicated, due to the nature of implementation of spatial domains in GIS. However, an additional attribute needs to be incorporated into the Gu-relation, which refers to the specific spatial domain. Formations (FO), as well representations of geologic units ( $\mathrm{Gu}$ ) are related to ages (AAge) for the reason that not each mapped unit is officially assigned to a stratigraphic code. This way, geometries can be queried directly via their age. In this example, all units (their IDs) are queried whose ages are between $60 \mathrm{Ma}$ and $70 \mathrm{Ma}$.

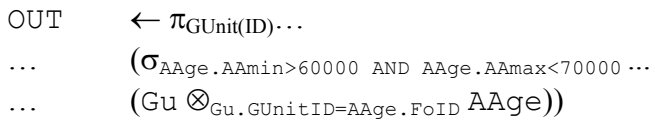

This translates to the following query:

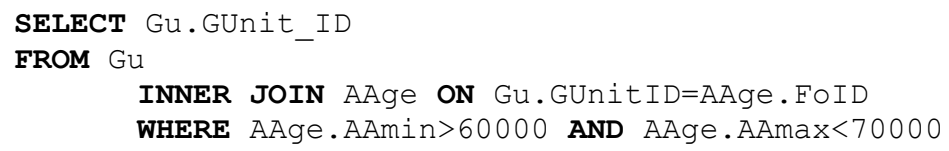

Chronostratigraphic relationships are joined hierarchically and related to absolute ages and geometry. Depending on the expected output, either ages or geometric identifiers are returned for further processing.

\section{Summary and Outlook}

A time-integrative data model can be a simple piece of SQL code, or it can be designed interactively using ArcGIS Model Builder tools - the choice depends on the actual needs within a given project. The usability of queries, however, requires some additional thoughts. While the implementation of geologic time is in principle possible, querying data 
requires a more convenient design, and this can only be accomplished by dedicated GUI design with dedicated query variables and operators $(</ \leq / \geq \mid=/>)$.

Geologic units and their core attributes can be rigorously modeled for GIS applications as long as time remains an attribute, and as long higher-level normalization and operational performance are of secondary importance. This has been shown in a number of contributions on geologic data models in the past. The relationships between relative and absolute time concepts as defined through basic stratigraphy cannot satisfactorily be modeled as a simple model attribute, due to the inherent non-temporal approach in modern GIS environments, and it is probably not possible to provide a generic time architecture that allows being employed within each and every research and application field. Demands for time integration have today been answered by providing, e.g., time-step analysis functions in commercial GIS suites (e.g., ESRI's tracking analyst), which usually build upon a single time attribute. Consequently, attempts to generically model time on the attribute level leads to either highly complex data models, to maintenance problems (DB anomalies), or to query limitations.

The most important aspect of time queries in geology are related to (1) cross-correlating different units across different definitions (standards) of geologic time boundaries, (2) cross-correlating sequences of materials deposited within a given time range, and (3) building and querying a relative sequence with non-temporal data. The efficiency (in terms of querying and managing) and extensibility of a geologic data model severely suffers if the design does not cope with temporal changes and time constraints. It has been shown, that a generic time-integrative geologic data model can be designed without losing control over the database's consistency and future extension requirements. Object-relational capabilities, such as subtype definitions, in modern DBMS and GIS concepts do help to maintain these capabilities if time is modelled on the attribute level. If implemented for the use with standard GIS, the complexity of queries is constrained only by the user interface definition, i.e., the external layer. It cannot be expected from any mapper to operationally formulate queries, and it is therefore envisaged to provide a customizable set of pre-defined queries according to the expected results in which the user can easily select variables and operators.

\section{References}

Claramunt, C. \& Theriault, M. (1995), Managing Time in GIS: An Event-Oriented Approach. In: Clifford, J. \& TuZHiLin, A. (Eds.), Recent Advances in Temporal Databases: Proceedings of the International Workshop on Temporal Databases, Springer, Zürich, 23-42.

Couvering, J. A. \& OgG, J. G. (2007), The Future of the Past: Geological Time in the Digital Age. Stratigraphy, 4, 253-257.

CoX, S. J. D. \& RichaRD, S. M. (2015), A geologic timescale ontology and service. Earth Science Informatics, 8 (1), 5-19.

Date, C. J., Darwen, H. \& Lorentzos, N. A. (2003), Temporal Data and the Relational Model: A Detailed Investigation into the Application of Internal and Relation Theory to the Problem of Temporal Database Management. Morgan Kaufmann, Amsterdam, xxii, $424 \mathrm{p}$. 
Gould, S. J. (1987), Time's Arrow and Time's Cycle. Myth and Metaphor in the Discovery of Geological Time. Harvard University Press, Cambridge, US, xv, $217 \mathrm{p}$.

Gradstein, F. M., OgG, J. G., Schmitz, M. D. \& OgG, G. M. (2012), The Geologic Time Scale 2012. Elsevier, Amsterdam, 1176 p.

LANGRAN, G. (1992), Time in Geographic Information Systems, Technical Issues in Geographic Information Systems. Taylor \& Francis, London, xii, 190 p.

LE, Y. \& USERY, E. L. (2009), Adding Time to GIS. In: MADDEN, M. (Ed.), Manual of Geographic Information Systems, chap. 19.The American Society for Photogrammetry and Remote Sensing (ASPRS), Bethesda, 311-332.

NACSN (2005), Code of Stratigraphic Nomenclature. The American Association of Petroleum Geologists Bulletin, 89, 1547-1591.

OgG, J. G., OGG, G. \& Gradstein, F. M. (2008), The Concise Geologic Time Scale. Cambridge University Press, Cambridge, vi, $177 \mathrm{p}$.

OTT, T. \& SWIACZNY, F. (2001), Time-Integrative Geographic Information Systems. Manag. and Analysis of Spatio-Temporal Data. Springer, Berlin, 3rd Ed., xiv, $234 \mathrm{p}$

Peuquet, D. J. (1999), Time in GIS and Geographical Databases. In: Longley, P. A., Goodchild, M. F., MAguire, D. J. \& Rhind, D. W. (Eds.), Geographical Information Systems, chap. 1. Wiley, Chichester, UK, 2nd Ed., 91-103.

Pred, A. (1977), The Choreography of Existence: Comments on Hägerstrand's Time Geography and its Usefulness. Economic Geography, 53, 207-221.

RAPER, J. (2005), Multidimensional Geographic Information Science. Taylor \& Francis, London, xxi, $317 \mathrm{p}$.

SAlVADOR, A. (Ed.) (1994), International Stratigraphic Guide. Geological Society of America and the International Union of Geological Sciences (IUGS), Boulder, 2nd Ed., xix, $223 \mathrm{p}$.

Soller, D. R. \& Berg, T. M. (2003), The National Geologic Map Database Project: Overview and Progress, in: Digital Mapping Techniques '03 - Workshop Proceedings, Open-File Report 03-471, U.S. Geological Survey, 57-77.

Soller, D. R. \& Stamm, N. R. (2008), The National Geologic Map Database Project 2008 - Report of Progress. In: Soller, D. R. (Ed.), Digital Mapping Techniques '08 Workshop Proceedings, Open-File Report 2009-1298, U.S. Geological Survey.

STENO, N. (1917), De solido intra solidum naturaliter contento dissertationis prodromus, translation 1917 (The Macmillan Company): The prodromus of Nicolaus Steno's dissertation concerning a solid body enclosed by process of nature within a solid. Florence, $115 \mathrm{p}$.

ThrifT, N. (1977), An Introduction to Time-Geography. Concepts and Techniques in Modern Geography (CATMOG). Geo Abstracts Ltd., Norwich, UK, iii, 37 p.

WACHOWICZ, M. (2003), Object-Oriented Design for Temporal GIS, Research Monographs in Geographical Information Systems. Taylor \& Francis, London, xiv, 118 p. 